\title{
Acerca del devenir de los paradigmas epistemológicos en Pedagogía Social y su impacto en el surgimiento de un pensamiento pedagógico latinoamericano
}

\author{
About the development of epistemological paradigms \\ in Social Pedagogy and its impact on the emergence \\ of a Latin American pedagogical thought
}

María Paula Juárez ${ }^{1}$

\begin{abstract}
Resumen: El artículo tiene el propósito de realizar un recorrido en clave de lectura sociohistórica educativa en relación a la evolución de la Pedagogía Social situada en un contexto global y local. Se parte de considerar el impacto que los paradigmas epistemológicos subyacentes a su desarrollo han generado en ella en un devenir dinámico entendido como proceso de cambio continuo. El análisis se orienta a comprender las características que presentó dicho desarrollo considerando los paradigmas positivista y sociocrítico como grandes corrientes de pensamiento que movilizaron las Ciencias Sociales a lo largo de la historia y -en particular- la influencia que han tenido en el campo pedagógico, donde se destaca la configuración y el escenario de surgimiento de una pedagogía propiamente latinoamericana, entendida como un paradigma emergente de nuestra región.
\end{abstract}

Palabras clave: educación, epistemología, pensamiento, América Latina, sentido crítico.

\footnotetext{
${ }^{1}$ Doctora en Psicología. Magíster en Ciencias Sociales. Magíster en Iniciación a la Investigación en Psicología de la Comunicación, Interacción Social y Desarrollo Humano. Licenciada en Psicopedagogía. Docente de la Universidad Nacional de Río Cuarto. Investigadora Asistente del Consejo Nacional de Investigaciones Científicas y Técnicas (CONICET). Río Cuarto, Córdoba, Argentina. Correo electrónico: mpaulajuarez@gmail.com.
}

Diālagos Pedagágicas. ISSN en línea: 2524-9274.

Año XVIII, No 35, abril - septiembre 2020. Pág. 1-23.

DOI: http://dx.doi.org/10.22529/dp.2020.18(35)01 / Recibido: 2-11-2018 / Aprobado: 17-02-2020.

Artículo publicado bajo Licencia Creative Commons Atribución-NoComercial-SinDerivar. (c) Universidad Católica de Córdoba. 
Abstract: The purpose of this article is to carry out a journey in a socio-historicaleducational key in relation to the evolution of Social Pedagogy within a global and local context. It starts by considering the impact that the epistemological paradigms underlying its development have generated in it in a dynamics understood as a process of continuous change. The analysis is oriented to understand the characteristics of this development considering the positivist and sociocritical paradigms as great schools of thought that mobilized the Social Sciences throughout history, and -in particular-the influence they have had in the pedagogical field, highlighting the configuration and the scenario of the emergence of a properly Latin American pedagogy, understood as an emerging paradigm of our region.

Keywords: education, epistemology, thinking, Latin America, critical sense.

\section{Presentación}

Este trabajo parte de la pretensión de realizar un recorrido en clave de lectura sociohistórica en relación a la evolución que ha tenido la Pedagogía Social en un escenario global y local, a partir del impacto que los paradigmas epistemológicos subyacentes a su desarrollo han generado, y la emergencia, a partir de ese devenir, de un paradigma pedagógico propiamente latinoamericano.

Desde un análisis crítico y reflexivo, se desarrollarán los paradigmas contrapuestos de base positivista y sociocrítica, se analizarán sus núcleos conceptuales básicos, lo que permitirá situar el escenario que le ha dado emergencia a una Pedagogía Social Latinoamericana.

El educador popular argentino Marcelo Krichesky (2011), parte de la idea que plantea que si bien la Pedagogía Social es un saber que tiene por objeto a la educación social en Europa y a la educación popular en América Latina, no refiere a lo mismo en ambos contextos. El autor señala que la Pedagogía Social ligada a la educación social y aquella vinculada a la educación popular "tienen historias e itinerarios de producción diferentes. Constituyen plataformas de pensamiento e intervención educativa, con enfoques disciplinares y prácticos diversos, que responden a paradigmas y contextos sociohistóricos distantes entre sí" (2011, p. 56)².

\footnotetext{
2 Es necesario atender, con cierta relatividad, a esta afirmación del autor, dado que no es posible desconocer los procesos que se han realizado en diversos países de nuestra región latinoamericana con relación al desarrollo de profesionalidades y prácticas bajo el nombre de "educación social" como objeto de estudio de la "pedagogía social". En este sentido, una recontextualización de las características de la Pedagogía Social en nuestro continente debe atender tanto a la educación popular como a la educación social como nutrientes de una pedagogía critica en Latinoamérica. Se desarrollan, particularmente desde el movimiento de la educación social, experiencias de formación en Uruguay (Espiga, López \& Morales, 2012); en Argentina (Borzese, Costas \& Wanger, 2011) en las provincias de Buenos Aires (https://educacionute.org/tecnicaturasuperior-en-pedagogia-y-educacion-social-con-orientacion-en-derechos-humanos-ciclo-lectivo-2017/) y Mendoza (http://educacion.uncuyo.edu.ar/estudios/carrera/tecnicatura-universitaria-en-educacion-social); alternativas de formación y un movimiento de educación social, cuyo objeto es la Pedagogía Social en Bolivia (https://www.ucbcba.edu.bo/pedagogia-social/); dos proyectos de ley que actualmente se dinamizan para reglamentar la educación social en Brasil y un movimiento organizado por universidades posicionadas desde la educación social en ese país (Monteiro Machado, 2011), así como iniciativas de trabajos colectivos que integran los mencionados países junto con México, quienes desarrollan sistemáticamente congresos y publicaciones sobre el campo y las prácticas de la Pedagogía Social y la Educación Social.
} 
Se analizarán estos aspectos de acuerdo a los paradigmas epistemológicos subyacentes. Luego, se reflexionará sobre ellos y se los interpelará también. Para ello, el artículo se organiza en tres apartados: inicialmente, se identificarán las características y núcleos conceptuales básicos del paradigma positivista para reconocer su influencia en el campo pedagógico y en la realidad socioeducativa con una reseña del escenario europeo, $y$, en nuestra región, se puntualizará sobre lo sucedido en Argentina. Seguidamente, se analizará el paradigma sociocrítico, sus constructos epistemológicos y pedagógicos y su impacto en la Pedagogía Social, para -finalmente- reflexionar, en relación al contexto de emergencia, particularidades y potencialidades que asume el paradigma educativo latinoamericano en los tiempos que corren.

\section{Punto de partida: breves consideraciones sobre el paradigma positivista en la Pedagogía Social}

Jamás puedes llegar allá partiendo de allá. Sólo se puede reconocer la existencia de un allá porque hay un acá, $y$ viceversa. Uno es el contrario del otro, el que ilumina al otro, el que me dice que el otro existe. Tú, por ejemplo, no podrías llegar a Sao Paulo partiendo de Sao Paulo sino partiendo de Managua, de la misma manera que tú sólo puedes llegar a Managua partiendo de Sao Paulo y no partiendo de Managua.

(Freire en Torres, 1985, p. 121)

Se parte de esta cita del pedagogo brasileño Paulo Freire para representar la dinámica que implica la lógica dialéctica en la comprensión de los opuestos. Atendiendo a ello, se considera que solo es posible llegar a la construcción de un conocimiento profundo y significativo de una Pedagogía Social de base sociocrítica latinoamericana si partimos de entender las características contextuales que le dieron emergencia, en reacción a qué escenario surge, lo que generó que esta perspectiva pueda conformarse y desarrollarse en la realidad.

Asimismo, abordar la cuestión de los paradigmas en pedagogía conduce necesariamente al tema de la epistemología y los posicionamientos.

En sentido estricto, se entiende la epistemología ${ }^{3}$ como el estudio de las condiciones de producción y de validación del conocimiento científico (Klimovsky, 1994;

\footnotetext{
3 Vélez (2002) considera que esta forma de comprender la epistemología se realiza desde un "sentido restringido" y se la define como una disciplina que tiene como objeto de estudio las condiciones de producción de las teorías científicas, los modos en que estas se elaboran, el lenguaje y la estructura con que se exponen sus principios y la lógica de sus enunciados. Asimismo, plantea un "sentido amplio" de la epistemología, que implica el estudio sistemático de las condiciones de producción, estructura y justificación de todas las formas que asume el conocimiento humano. Díaz (2007, p. 4) propone una "epistemología ampliada a lo político social" la que supone considerar el núcleo más duro de la ciencia en el entretejido de fuerzas en el que se produce y se desarrolla, atendiendo a luchas de poder, factores económicos, connotaciones éticas, afecciones, pasiones, idearios colectivos, intereses personales $y$ pluralidad de nutrientes vinculados al éxito o fracaso de las teorías.
} 
Klimovsky \& Hidalgo, 1998). A lo largo de la historia de la humanidad, ello ha implicado diferentes posturas a la hora de conocer la realidad $y$, de acuerdo a la posición que los hombres y mujeres asumen en su tiempo histórico, ha sido el modo de entender cómo se construye el conocimiento, qué es la educación y qué es la Pedagogía Social.

Domingo Bazán Campos (2008) plantea que el aporte más destacado en el tema de los paradigmas ha sido el de Thomas Kuhn, quien -en 1962- publicó su libro La estructura de las revoluciones científicas. A partir de sus planteos, comienza a emplearse el término paradigma para apelar a los modos de concebir, abordar y conocer la realidad que posee un grupo de científicos y pensadores en un momento particular de la historia. Se considera que el paradigma vigente permite comprender, resolver y ofrecer respuestas a todo lo planteado: no obstante, cuando emergen nuevos problemas que no pueden ser resueltos por este paradigma, cuando ya no ofrece nuevas respuestas a los interrogantes de la época, se generan anomalías y la ciencia normal entra en crisis. Es así como el paradigma comienza a perder vigencia y queda obsoleto. Esto significa que el paradigma anterior se desecha y un nuevo paradigma -denominado alternativo- surge y desarrolla nuevos principios, metodologías y visiones del mundo que le permiten resolver los problemas planteados, responder a nuevas preguntas, conocer la realidad y, así, avanzar en el desarrollo de la ciencia y la producción de conocimiento (Vélez, 2002).

Desde un sentido amplio (Vélez, 2002), Quintar (2018) considera que la epistemología remite a una cuestión de mirada de la vida, de comprensión del mundo, de mirada de la realidad. Refiere que, de acuerdo a cómo los sujetos ven la vida, se relacionan y generan prácticas, representaciones y formas de organizar los esquemas de pensamiento desde los cuales se conoce el mundo.

Bazán Campos (2008) advierte que, en el campo de la Pedagogía, no se da una modificación de paradigmas de corte lineal (es decir, cuando un nuevo paradigma emerge, el anterior desaparece), sino que lo que ocurre, en el escenario de la práctica educativa real y concreta, es que los viejos paradigmas coexisten junto a los nuevos, se dinamizan en los contextos reales de las instituciones educativas, se corporizan en los quehaceres de los equipos docentes, incluso conviven de manera contradictoria, muchas veces, en las cosmovisiones y prácticas de un mismo docente. Se genera así, dice el autor, un escenario "multiparadigmático" que permite reconocer la presencia de un paradigma dominante y de un paradigma alternativo en Pedagogía.

Asimismo, el autor plantea que, en Ciencias Sociales, se acepta que existen al menos- dos formas de entender la ciencia: una positivista-naturalista, hipotética-deductiva, tecno-científica, empírica-analítica, dominante y otra forma a la que suele denominarse paradigma alternativo, interpretativo, hermenéutico, comprensivo, crítico-social.

Como se planteaba inicialmente, para llegar a la comprensión del paradigma sociocrítico es necesario transitar la comprensión previa del contexto que le dio emergencia, entender en reacción a qué surge. Es así que el paradigma sociocrítico se configura como alternativa al paradigma positivista que nace en Europa entre 
Ios siglos XVII al XIX con representantes como Auguste Comte, Herbert Spencer y Émile Durkheim, quienes -sustentados en el racionalismo e iluminismo filosóficoponen énfasis en la búsqueda de objetividad y de conocimientos universales y plantean que la realidad es única y que puede llegarse a su conocimiento solo por el método científico. Este método para construir conocimiento es el denominado hipotético-deductivo, propio de las Ciencias Naturales, y trabaja con hipótesis comprobables a través de la observación y experimentación de los fenómenos.

Vogliotti (2016) considera que el conocimiento es sobre los fenómenos observables, se concibe como una copia fiel de la realidad y se construye como verdad universal y objetiva que se traspasa de generación en generación. Se caracteriza por ser objetivo, sistemático, comprobable, medible, concreto, controlable, empírico, lógico y cuantificable. Busca la causa de los fenómenos observables para formular generalizaciones o leyes a partir de las regularidades de los fenómenos. En lo que refiere a la relación que el sujeto establece con el objeto de conocimiento, aquel accede a la realidad a través de sus sentidos, va descubriendo los conocimientos de manera racional y se despoja de toda apreciación subjetiva. La realidad se concibe como aquella que está afuera del sujeto, constituida por hechos y donde solo lo que se ve existe. Esta realidad se caracteriza por ser única, objetiva, está dada, es independiente y se rige por regularidades que pueden ser descubiertas por los sujetos.

Coincidiendo con ello, Quintar (2018) plantea que el paradigma positivista mira el mundo de la vida desde afuera, establece una relación de externalidad con la realidad desde un pensamiento desarraigado de ella, procede de explicaciones sustentadas en criterios de verdad que orientan y se aplican a la realidad desde una lógica clasificatoria que escinde el mundo de la vida, desde un pensamiento lineal y causal que prescribe cómo es esa realidad concebida como un objeto.

En este escenario, Vogliotti (2016) plantea que la sociedad es una entidad que funciona de manera independiente, dividida y fragmentada, que se mantiene por procesos impersonales que funcionan como leyes. La sociedad es comprendida desde una perspectiva funcionalista en tanto se la concibe como un todo organizado basado en la división del trabajo, donde cada una de las partes que la componen, es decir hombres y mujeres, deben prepararse para ocupar una posición o función definida dentro de la estructura social.

El artículo establece que el positivismo acompañó la organización política y económica de los Estados Nacionales desde las premisas del liberalismo, una fe ciega en la ciencia y el progreso capitalista. En sintonía con ello, Quintar (2018) considera que esta tradición de pensamiento surge en el capitalismo y le otorga sustento desde una lógica civilizatoria, cuyo centro no fue solo el mercado, sino todo un sistema clasificatorio, jerárquico y patriarcal.

La autora plantea que este pensamiento abreva en una larga tradición donde se encuentra el positivismo como perspectiva inicial, seguido por los desarrollos del neopositivismo y el falsacionismo. Puntualmente, señala el neopositivismo lógico iniciado con la Escuela de Viena (1922), movimiento que tuvo distintas ramificaciones en Estados Unidos y Gran Bretaña, algunas de las cuales permanecieron 
ligadas a la denominación original de neopositivismo o positivismo lógico y otras se vincularon a un llamado empirismo lógico.

En el campo pedagógico, el positivismo implicó el surgimiento de una pedagogía entendida como una tecnología, sustentada en una racionalidad instrumental basada en la idea de conocer para hacer. Se trató de una pedagogía normativoprescriptiva, que se dinamizaba en el hecho de que la teoría pedagógica determinaba lo que había que hacer en la práctica educativa. La intención de esta pedagogía era formar un sujeto pragmático, productivo, racional y tecnológico, que se adaptara a las demandas del mercado en una sociedad industrial capitalista y que se incorporara a ella con éxito en la medida que adquiría habilidades, destrezas, hábitos y competencias tecnológicas (Vogliotti, 2016). Se promovió así un modelo tecnológico -también Ilamado Pedagogía Tecnicista- y se desarrollaron pedagogías experimentales o pedagogías psicométricas basadas en el uso de tests e instrumentos para medir aptitudes.

En este sentido, la pedagogía como teoría estaba sometida a las mismas normas de teorización propias de las Ciencias Naturales y buscaba fundamentación en la Biología, la Psicología Conductista y la Sociología Funcionalista.

Quintar (2018) considera que -desde el positivismo- la Pedagogía fue concebida como ciencia que se configuró atravesada por un capitalismo moderno que se movilizó por los intereses del mercado y se orientó a acomodar a los sujetos a determinados lugares de acuerdo a las demandas que el mismo mercado generaba.

Algunos constructos pedagógicos centrales que pueden identificarse en una pedagogía de base positivista son los siguientes: La concepción de escuelas como sistemas sociales naturales que emergen para satisfacer las necesidades del individuo y la sociedad capitalista, orientadas hacia un fin, donde las conductas de los sujetos son determinadas por normas y por el ejercicio de una autoridad legítima. Hay una nula vinculación de la escuela con las problemáticas sociales de la realidad. La escuela aparece como la institución básica que posee el Estado, cuya tarea es preparar intelectual y moralmente a los alumnos para asumir una posición en la sociedad y que estos respondan a sus intereses. Se trata de un medio de transformación ideológica y cultural para formar a los jóvenes. En la relación educativa, el docente se presenta como el que sabe, el que piensa y transmite en forma acabada los conocimientos y el alumno, una tabula rasa que carece de conocimientos y recepta esa información y se le exige la memorización de la información que el docente narra y expone. Se trata de una relación mediada por la programación y la tecnología. Docente y alumnos son reducidos a meros transmisores y consumidores, donde el protagonismo del acto educativo lo asumen los programas, medios y objetivos. No obstante, esta relación está basada en el predominio de la autoridad del docente, quien exige una actitud receptiva y pasiva del estudiante, cuya obediencia es la principal virtud (Barrios, 2011). El conocimiento se centra en el objeto y el sujeto debe descubrirlo, dado que ya existe en la realidad. El conocimiento se valida por el criterio correspondentista, es decir, acciones sistemáticas y ordenadas en donde la teoría se aplica a la práctica. Para el positivismo, el interés por el conocimiento es técnico-instrumental o práctico y se vincula a 
aplicar los conceptos en la realidad de manera unidireccional. Como contenido de la enseñanza, el conocimiento consiste en saberes y valores acumulados por las generaciones adultas que se transmiten a los alumnos como verdades acabadas, disociados de las experiencias de los alumnos y de las realidades sociales ( $\mathrm{Ba}$ rrios, 2011). El currículo se caracterizó por prescribir lo que se enseñaba y se aprendía en la escuela. Se fijaban metas explícitas desde objetivos que perseguían conductas observables, medibles y controlables a través de la evaluación y su finalidad era la formación de recursos humanos para la producción. Su organización era fragmentada, con nulos espacios para integrar los conocimientos a las problemáticas sociales de la realidad. Los contenidos se seleccionaban desde un criterio pragmático y de aplicación a la vida cotidiana y futuro desempeño laboral.

Esta tendencia pedagógica no consideró trabajar sobre los procesos de aprendizaje de los alumnos, ya que no atiende a la manera y las formas en que se va configurando en ellos la construcción de conocimiento, sino que se centra en la evaluación del aprendizaje con énfasis en el resultado, apartando su foco de los procesos de análisis y razonamiento de los sujetos (Barrios, 2011).

\subsection{Acerca del impacto de este paradigma en la realidad de una Pedagogía Social}

Este apartado considerará brevemente el impacto del paradigma positivista en la Pedagogía Social europea y en la pedagogía de nuestra región latinoamericana, particularmente en el caso de Argentina.

Los orígenes de la Pedagogía Social se sitúan en un enclave propiamente europeo en los siglos XVIII y XIX y logró su desarrollo en Alemania, España, Italia, Bélgica, Francia e Inglaterra (Pérez Serrano, 2002; Núñez, 2007; Monteiro Machado, 2011; Caride, Gradaílle \& Caballo, 2015, entre otros). En general, los autores coinciden en considerar que los factores que dieron origen a la Pedagogía Social fueron una revolución industrial generadora de marginación, "inadaptación" y desamparo en la sociedad, profundas transformaciones y fenómenos de masificación urbana, desplazamiento masivo del campo a la ciudad, proletarización del campesinado, pobreza, escenario de carencia y necesidad en la posguerra. Ya a partir de 1970, se incorpora en los currículos universitarios, se desarrollan investigaciones, se amplía su objeto y concepto atendiendo a todas las personas, grupos, comunidades en situación de necesidad y en distintas situaciones existenciales -más allá de la escuela- (Pérez Serrano, 2002).

A pesar de que en la actualidad pueden reconocerse distintas vertientes en la Pedagogía Social europea y que los desarrollos en los últimos años dan cuenta, incluso, de una vertiente crítica en este campo (Núñez, 2007), Krichesky (2011) considera que la perspectiva hegemónica en Pedagogía Social en los países centrales ha estado sustentada en las tradiciones del pensamiento funcionalista, basada en principios moralizadores, higienistas y asistencialistas, que se configuró como una tradición ligada al control social de la población a través de la pedagogía. Esta tendencia puede reconocerse en estudios y desarrollos en una Pedagogía Social europea de base positivista, caracterizada por la intencionalidad de 
mantenimiento del orden social vigente, la idea de "adaptar" -claramente explicitada en distintos trabajos- al sujeto a una sociedad industrial en funcionamiento. Los planteos de sus precursores y representantes (desde los inicios e, incluso algunos, hasta nuestros días) expresan la idea de "prevención de aquello indeseable", la idea de "recuperación de individuos" con "conductas desviadas", consideradas "conductas de riesgo", "peligrosas", proclives a la realización de actos delictivos, con "dificultades de atención y adaptación sociocultural". Se trata de una pedagogía orientada a la infancia y la juventud que evidencia una perspectiva conservadora que, aun actualmente, coexiste junto con otras perspectivas.

En la región latinoamericana, particularmente en Argentina, si se intenta vincular el impacto que el positivismo ha dejado en la pedagogía, es la figura de Domingo Faustino Sarmiento (1811-1888) la que ha dejado huellas imborrables en la memoria histórica y educativa, al promover un proyecto de país asociado al progreso europeo y norteamericano. Para ello, importó de esas tierras ideas pedagógicas que resultaron en una educación que proponía un conocimiento individual, aislado, enciclopédico, de repetición, donde los contenidos eran "universales" y dejaban afuera los saberes de las culturas populares, y planteó sus ideas desde el lema que va dar título a su obra, Civilización y barbarie en las pampas argentinas, el cual influyó profundamente en la visión de una realidad fragmentada. Su modelo pedagógico triunfa asociado al proyecto político que conducen presidentes como Mitre, el propio Sarmiento y Roca, sustentado en la idea de organización nacional desde una pedagogía de raigambre liberal y positivista, funcional a las demandas y necesidades de un sistema capitalista basado en un modelo agroexportador. Al hablar de pedagogías latinoamericanas, la figura de Sarmiento es una referencia obligada, dado que representa una pedagogía oficial y hegemónica, una pedagogía que ha dejado marcas imborrables en las prácticas escolares y educativas argentinas, lo que llevó a Puiggrós $(1990 ; 2010 ; 2017)$ a incluirla dentro de las denominadas "pedagogías del olvido", así como es posible incluirla en la categoría de reflexión que Segato (2018) propone desde las "pedagogías de la crueldad".

\section{Aproximaciones al paradigma sociocrítico y su concepción en Pedagogía Social}

Como antítesis a este paradigma naturalista y a los impactos sociales generados por él y trascendiendo la mirada hermenéutica de los desarrollos fundamentales que implicó el paradigma interpretativo y simbólico para las Ciencias Sociales, desde los inicios en el siglo XVII, pero fundamentalmente en el siglo XX, se desarrolla un paradigma sociocrítico a través de la llamada Escuela de Frankfurt en Alemania (1923).

Esta corriente de pensamiento se funda a partir de la iniciativa de un grupo de pensadores (filósofos, politólogos, economistas, sociólogos, psicólogos, comunicadores) que comienzan a sistematizar el pensamiento crítico. Sus principales representantes son Theodor Adorno, Herbert Marcuse, Max Hortheimer, Walter Benjamin, Erich Fromm (entre otros, de la primera generación de desarrollos) y 
Jürgen Habermas (quien es el más destacado de una segunda generación de desarrollo de este pensamiento). Durante la Segunda Guerra Mundial y bajo el régimen nazi, la mayoría de sus miembros son exiliados en EE.UU., por su condición de judíos, país donde continúan sus desarrollos. Vogliotti $(2015,2016)$ plantea que su pensamiento, desde un enfoque sociocrítico, surge como una fuerte reacción y crítica ante:

- El sistema capitalista y lo que estaba generando en las sociedades, desde la alienación del sujeto, las relaciones de dominación y explotación, la promoción de valores sustentados en el individualismo y la competencia, la generación de diferencias sociales injustas, desigualdad, exclusión y marginación.

- El enfoque del positivismo, por su reduccionismo en el tratamiento de las cosas, por su visión técnica y mecanicista de la ciencia y por dar sustento teórico al capitalismo desde su racionalidad instrumental.

La Teoría Social-Crítica se fundamenta en una filosofía para la emancipación y busca sus bases teóricas en la Historia, la Sociología y el Psicoanálisis de Freud y realiza una revisión crítica de las teorías de Marx y Hegel. Propone construir una realidad humana más justa y considerar una dimensión múltiple de enfoques para abordar problemas que hay que transformar.

Desde este paradigma, se concibe que la realidad es construida por los sujetos desde sus significados y valoraciones, situados en un contexto histórico-social determinado. La realidad es inconclusa, inacabada, histórica, atravesada por dimensiones políticas, sociales, culturales y económicas. Pero también es compleja, contradictoria, dinámica, cambiante y cambiable. Las situaciones de la realidad no pueden ser entendidas al margen del contexto y sus condicionantes. La realidad misma es producto de la acción de los hombres y mujeres, por lo tanto, transformar esa realidad es su tarea a través de la desnaturalización (Vogliotti, 2016). Esta consiste en cuestionar, interrogar, preguntar y romper con la visión ingenua de la realidad social que da por naturales los fenómenos sociales para cambiar ese conocimiento por otro crítico que explique las situaciones injustas y contradictorias (Freire, 1973).

El ser humano es entendido como un sujeto crítico, social, que es con otros, inacabado, inconcluso, consciente de sí y del mundo, es constructor de la historia y está movido por el "ser más" del humanismo como una vocación por la cual los seres humanos asumen su liberación desde la comunión con los otros (Freire, 1973, 1993) y no por "el tener más" del capitalismo como búsqueda centrada en el individualismo egoísta que resulta en una forma de "ser menos" (Freire, 1973, 1993).

Desde esta perspectiva, las acciones y conocimientos del sujeto no son neutras, implican un posicionamiento e intereses en torno a la realidad.

Quintar (2018) considera que, en el paradigma sociocrítico, la mirada es desde la internalidad, la cual -al ser parte de la misma realidad- se relaciona con el mundo de la vida y los sujetos en coordenadas de presente, desde un pensamiento histórico situado en la realidad. Desde esta perspectiva, no hay verdades dadas, sino verdades historizadas que se construyen históricamente por los sujetos, que no buscan explicar, sino comprender desde un movimiento de pensamien- 
to y lectura constante que implica recurrentemente el distanciarse y aproximarse al mundo de la vida. Aquí la crítica emerge como un modo de construir conocimiento en el presente, como un modo de pensar, como una crítica histórica, que configura el mundo de la vida en un verdadero activador en la construcción de conocimiento.

En relación a los núcleos conceptuales básicos de este paradigma, podemos diferenciar entre los constructos epistemológicos, vinculados a la manera de conocer desde esta perspectiva, y los constructos propiamente pedagógicos, aquellos que emergen del impacto de esta teoría en la educación.

Respecto a los constructos epistemológicos, se considerará la concepción de conocimiento, método, investigación y teoría.

El conocimiento es entendido como una construcción sociocrítica, contextualizada, intersubjetiva, es humanizador y liberador. Vogliotti (2016) entiende que es crítico en tanto pone al descubierto intereses y valores, que subyacen en las prácticas; esto significa que el conocimiento no es neutral, es interesado, no hay acción social o conocimiento que sea ideológicamente o políticamente neutro. En este sentido, se reconoce la dimensión valorativa del conocimiento porque este siempre está orientado por intereses, ya sean de la hegemonía o de la emancipación. El conocimiento es desvelador del orden social existente y de las relaciones de poder. Es la construcción de conocimiento de la realidad la que posibilita la toma de conciencia de la propia situacionalidad en el mundo, es decir, el paso de un conocimiento ingenuo a un conocimiento crítico de la propia realidad. Se concibe que los sujetos no construyen conocimientos de manera solitaria o aislada, sino a través de un proceso de interacción de puntos de vista subjetivos con otros, orientados a promover cambios sustentados en la solidaridad y la justicia social. El conocimiento no es único ni determinado, sino que se caracteriza por ser discontinuo, de ruptura, dialéctico, utópico, combina un lenguaje de crítica (denuncia) y un lenguaje de posibilidad (anuncio) (Vogliotti, 2016).

El método que se utiliza para construir conocimiento es el método sociocrítico que implica un proceso de problematización de la realidad, de reconocimiento de las contradicciones; su intencionalidad es la concientización de los sujetos acerca de sus condiciones concretas de existencia, la visualización de las posibilidades de cambio y la generación de propuestas alternativas y su concreción. Las metodologías que se emplean involucran la praxis concreta y son las siguientes: la investigaciónacción, la investigación participante y la investigación colaborativa (Vogliotti, 2016).

Desde esta perspectiva, la investigación es una práctica social comprometida con el cambio y la liberación de las situaciones de dominación, implica el involucramiento de los sujetos investigadores e investigados en la propia realidad; está basada en la comunicación intersubjetiva y supone la explicación y la transformación de las situaciones estudiadas. En este sentido, la relación entre sujeto y objeto es dialéctica y dialógica, no existe uno sin el otro. La teoría es entendida como reflexión en y desde la acción; teoría y realidad mantienen una constante tensión dialéctica; teoría y práctica son indisociables. La práctica es teoría en acción (Vogliotti, 2016). 
En el campo pedagógico, este paradigma se dinamizó desde lo que se define como Pedagogía Crítica, la cual es entendida como una praxis, en tanto implica una conjunción de la teoría con la práctica en una relación dialéctica. Es denominada pedagogía crítica, liberadora, problematizadora, crítico-reflexiva y plantea que la teoría pedagógica se va reconstruyendo contantemente en la práctica, desde una praxis transformadora y formadora de conciencia crítica (Vogliotti, 2001).

Los constructos pedagógicos centrales de la pedagogía crítica (Vogliotti, 2015, 2016) que se consideran a continuación se vinculan a la intencionalidad u objetivos de la pedagogía, la relación pedagógica, la construcción de conocimiento, las escuelas y el quehacer del educador.

La intencionalidad de la pedagogía crítica se refiere a la transformación radical de la sociedad, liberación o emancipación. Se trata de formar sujetos críticos, humanizados y emancipados, comprometidos con la justicia social. Por lo que esta pedagogía se configura desde la concepción del conocimiento como una construcción sociocrítica, contextualizada, intersubjetiva, objetivada, humanizadora y liberadora.

La relación pedagógica se sustenta en el encuentro dialógico, donde educador y educandos se orientan a la construcción de significados comunes desde los aportes de saberes que hace cada uno. Se trata de una relación horizontal en el plano dialógico; no obstante, en el plano de conocimiento, el educador debe ofrecer la novedad, un nuevo conocimiento que -a partir de los saberes de los que disponen los educandos- permita construir un nuevo conocimiento crítico y significativo de aquello que se conoce. Los saberes de los educandos son la plataforma adecuada para construir ese nuevo conocimiento crítico, profundo, reflexivo y superador.

Se conoce la realidad intersubjetivamente a través de su problematización; los sujetos no conocen solos o de manera aislada, sino que conocen en relación con otros hombres y mujeres mediatizados por su mundo, su realidad, y lo hacen desde metodologías dialógicas basadas en trabajos grupales y colaborativos, la integración de campos disciplinares y en relación a situaciones concretas de su contexto.

Las escuelas son construcciones sociales e históricas que propician la reproducción del orden social vigente, que genera opresión y explotación. En este sentido, se las entiende como ámbitos de conflictos, resistencias y protestas que, al mismo tiempo, permiten la toma de conciencia de la realidad injusta y contradictoria. En este sentido, son consideradas como escenarios de denuncia y anuncio: denuncian cualquier forma de dominación humana, la deshumanización, desenmascaran al conocimiento dominante, a los intereses hegemónicos, al pensamiento ingenuo, a los medios de comunicación, al discurso político desde un interés liberador y proponen alternativas superadoras con respecto a las contradicciones de la realidad y de la sociedad a través de proyectos colectivos orientados a una sociedad más justa y democrática.

El pedagogo-educador se guía por el propósito de formar personas dispuestas a trabajar para la transformación, la humanización, para la modificación del sentido que se atribuye a las cosas, al promover prácticas capaces de interpelar la 
propia situacionalidad en el mundo y evaluar y generar intervenciones innovadoras, desde el diálogo y la participación para la modificación de la realidad social. Como pedagogo, el educador reflexiona profundamente sobre sus propias prácticas educativas; como educador, el pedagogo no se queda en el plano de la reflexión, sino que dinamiza una praxis comprometida con acciones de cambio concretas y realizables. En este punto, resulta interesante el interrogante que plantea Bazán Campos (2008), dado que interpela respecto del impacto de los paradigmas en los pedagogos sociales. El autor se pregunta: ¿El pedagogo social se desempeñará regido por la idea de mantener el statu quo (positivismo) o por la convicción transformadora y democratizadora que ha mostrado la Pedagogía Social de corte crítico-social?

\subsection{Acerca del impacto del paradigma sociocrítico en la realidad de la Pe- dagogía Social europea y la emergencia de un pensamiento pedagógico latinoamericano propio}

En la conformación de la Pedagogía Social europea, la pedagoga española Gloria Pérez Serrano (2002, pp. 220-227) identifica una cuarta etapa, que abarca de 1950 a la actualidad, vinculada al desarrollo de una Pedagogía Social Crítica que recibe la influencia de Habermas con representantes como Mollenhauer, Giesecke, Hornstein, Thierach y Klafki, que desarrollaron el carácter reflexivo-crítico de la relación entre educación y estructura social, entendiendo que la Pedagogía Social parte de analizar las estructuras sociales para cambiarlas desde una educación emancipatoria orientada al análisis de los problemas de los sujetos y su solución.

Asimismo, impactaron, en Europa y en nuestro continente americano, los desarrollos que, desde el campo sociológico a la educación, representaron las "teorías critico-reproductivistas" que comprendieron que la función de la educación es la reproducción de la sociedad capitalista. Estas corrientes parten de entender que el sistema educativo ocupa un lugar esencial en la reproducción de la vida social, tanto por inculcar los principios de la ideología dominante como por ofrecer una oferta formativa diferencial, de acuerdo a los lugares a ocupar en los distintos puestos de trabajo del aparato productivo (Gvirtz, Grinberg \& Abregú, 2011).

Algunas de las teorías que mayor repercusión tuvieron fueron la "Teoría del sistema de enseñanza como violencia simbólica" de Bourdieu y Passeron; la "Teoría de la escuela como aparato ideológico del Estado" de Althusser y la "Teoría de la escuela dualista" elaborada por Baudelot y Establet. Respecto de ellas, el filósofo y educador brasileño Dermerval Saviani (1982) planteaba que, por un lado, desde el plano de la crítica, si bien estas teorías tuvieron el mérito de poner en evidencia el compromiso de la educación con los intereses dominantes al reproducir la ideología burguesa, por el otro, contribuyeron a diseminar un clima de pesimismo al no contener una propuesta pedagógica alternativa a la realidad denunciada. En América Latina, los desarrollos desde esta perspectiva tuvieron influencia en las décadas de 1960 y 1970; no obstante, en este escenario, se redobló la apuesta y no se permaneció solo en el plano de la denuncia. Quizás porque, des- 
de mucho antes, ya se apostaba por una praxis pedagógica en este suelo. Saviani (1982) plantea que la pregunta del momento fue ies posible encarar la escuela como una realidad histórica, susceptible de ser transformada? A lo que responde que una teoría crítica (que no sea reproductivista) solo podrá ser formulada desde el punto de vista de los intereses dominados.

Vinculado con ello, puede reconocerse, en la conformación de las pedagogías críticas latinoamericanas, el impacto de un contexto que, en el extremo sur, en países como Argentina y Chile, estuvo ligado a los procesos de lucha contra dictaduras militares y a los procesos de reconstrucción nacional desde la democratización. En Brasil, existía un escenario político social similar donde primaron campañas de alfabetización y postalfabetización de adultos campesinos. En países andinos como Bolivia y Perú, el acento estuvo puesto en la sabiduría y cultura de los pueblos originarios. En América Central, en países como Nicaragua, se vinculó a los procesos insurreccionales de la revolución popular sandinista y, en México, tiene su hito en las iniciativas independientes del partido de gobierno, como el movimiento de los zapatistas en Chiapas. En sintonía con ello, Quintar (2018) señala cómo el paradigma sociocrítico -como pensamiento histórico que se dinamizó desde la lógica de la lucha- generó aportes que emergieron desde situaciones de disconformidad, lo que dio lugar a una pedagogía entendida como construcción comunitaria de identidad. La autora reconoce aquí todo un movimiento de pedagogía crítica y educación popular que nace desde preocupaciones históricas en una corriente de pensamiento y teoría, entendida como construcciones vivas, y una pedagogía crítica que se instala en las tensiones, en las injusticias, en las incomodidades, movida desde la lógica de la indignación y la intencionalidad de recuperar las propias raíces.

Por su parte, Torres Carrillo (2009) y Krichesky (2011) coinciden en que una pedagogía de tinte social-crítica en nuestra región se vinculó a los movimientos contrahegemónicos al modelo liberal, identificados con una corriente más amplia de pensamiento y acción de raigambre latinoamericana, que se inicia en la década de los sesenta y llega hasta el presente. Forma parte de esta tradición la educación popular con destacados referentes como Paulo Freire, João Francisco de Souza, Carlos Núñez, entre otros.

Con respecto al pensamiento pedagógico latinoamericano, el educador popular colombiano Marcos Mejía (2017, p. 12) identifica un cuarto paradigma educativo ${ }^{4}$. Aunque si bien ha adquirido reconocimiento en la pedagogía crítica del norte, a través de pedagogos como Henry Giroux y Peter McLaren, aún no logra pleno reconocimiento en nuestra región.

El autor plantea que este paradigma educativo latinoamericano ha comenzado a desarrollarse desde las nacientes repúblicas hasta su auge (a fines de 1950 y durante el transcurso de las décadas de los sesenta y setenta del siglo anterior). Se trata de un pensamiento que, tomando inicialmente las corrientes críticas

\footnotetext{
${ }^{4}$ Mejía (2017) menciona la existencia de cuatro paradigmas educativos: 1) Paradigma alemán, 2) el francés, 3) el angloamericano y 4) el latinoamericano.
} 
euroamericanas, va trazando y delimitando las particularidades de un pensamiento propio, desde nuestros contextos, culturas, tradiciones. Surge así una educación popular y una pedagogía basada en el diálogo de saberes y la negociación cultural.

Este paradigma parte de una crítica a la modernidad (y a los efectos de una posmodernidad neoliberal) positivista, en cuanto su embestida de universalidad totalizante significa una "violencia epistemológica" (Mejía, 2011, p. 53) que niega otras formas de conocer fundadas en la cultura, el contexto, la diversidad y la diferencia y centra su propuesta en la necesidad de que la educación construya actores críticos, promotores de transformación de sus realidades. Mejía (2017) advierte cómo la teoría crítica, en sus diferentes vertientes euronorteamericanas, ha estado ligada a la denuncia y propuesta frente al capitalismo occidental, pero que esto no ha sido suficiente para explicar las particularidades de nuestra realidad. Por ello, necesita complementarse con las especificidades de las relaciones sociales gestadas en el proceso de la colonialidad ${ }^{5}$, que -en los países de la periferia- se ha manifestado como dominación, no solo en el ámbito del trabajo, sino en los ámbitos de la razón, el género y los saberes, lo que da forma a una subjetividad controlada. Es así como, a partir de la década de los cincuenta del siglo pasado y a lo largo de este nuevo siglo, se consolidó un pensamiento educativo crítico sustentado en construcciones conceptuales y prácticas como crítica a la forma de la cultura y la colonialidad. Se da así la construcción de un pensamiento propio, un movimiento intelectual con bases sociales y políticas desde América Latina orientado a constituir lo propio que busca diferenciarse de las formas eurocéntricas de saber.

Mejía plantea: "Varios pensadores críticos del mundo eurocéntrico construyen esa mirada homogeneizadora sobre los otros mundos (Asia, África, América), participando también de esa subalternización a las voces diferentes a las propias" (2011, p. 24). Relacionado con ello, podemos pensar cómo el pensamiento pedagógico latinoamericano desarrolló una crítica situada en nuestras tierras de la teoría social crítica europea, produciéndose así un "acercamiento-distancia con las teorías críticas euronorteamericanas a manera de tensión local-global" (Mejía, 2017,

\footnotetext{
5 Mejía (2011) diferencia entre colonialidad y colonialismo y entiende que de la colonialidad se sale mediante las luchas de independencia. Sin embargo, en el colonialismo, sigue operando una visión del mundo control de las mentes y los cuerpos, donde las instituciones educativas juegan un papel muy importante. El autor señala que el eurocentrismo no es una mirada cognitiva propia de los europeos, sino de todos los que hemos sido educados en los imaginarios y prácticas de las hegemonías de las instituciones educativas donde se ha naturalizado el imaginario cultural europeo, lo que invisibiliza y hace desaparecer las historias locales. Mignolo (2007) plantea cómo la "decolonialidad", entendida como un pensamiento que no se deja manejar por la lógica de la colonialidad, que emergió en la fundación misma de la modernidad/ colonialidad como su contrapartida con el pensamiento indígena y en el pensamiento afrocaribeño, continuó luego en Asia y África. El giro epistémico decolonial es una consecuencia de la instauración de la matriz colonial de poder, que Aníbal Quijano describe como la urgente crítica del paradigma europeo de la racionalidad/modernidad. El giro decolonial plantea la apertura y la libertad del pensamiento y de formas de vida-otras, el desprendimiento de la retórica de la modernidad y de su imaginario imperial. Es necesaria la decolonización epistemológica para dar paso a una nueva comunicación intercultural. El movimiento Sin Tierras en Brasil, los zapatistas en Chiapas, los movimientos indígenas y afros en Bolivia, Ecuador y Colombia, el Foro Social Mundial y el Foro Social de las Américas son ejemplo de un pensamiento decolonial planetario que no se limita a individuos.
} 
p. 25). En este sentido, el pedagogo argentino Enrique Bambozzi (2005) considera que el movimiento de la pedagogía del oprimido de Paulo Freire representa, en Latinoamérica, la apropiación crítica de las teorías reproductivistas europeas.

Se advierte así cómo el pensamiento pedagógico crítico latinoamericano ha bebido de la fuente de los desarrollos de la Teoría Social Crítica de la Escuela de Frankfurt (entre otras ${ }^{6}$ corrientes críticas y críticas reproductivistas), pero se configura como un pensamiento propio desde sus orígenes, donde se puede reconocer, desde la denominación de Mejía (2017, p. 74), como una de las corrientes críticas "de la singularidad y epistemes particulares". Esta se desarrolla en nuestro contexto, en el marco de un fecundo momento histórico de las Ciencias Sociales manifiesto en la dinamización de corrientes como la Teoría de la dependencia (Enzo Faletto, Fernando Cardoso, Theotonio Dos Santos, entre otros), la Teología de la liberación (Gustavo Gutiérrez, entre otros), Comunicación popular (Mario Kaplún), Investigación-acción participante (Orlando Fals Borda, Paulo Freire), Colonialidad del conocimiento (Aníbal Quijano), la Psicología Social (Ignacio MartínBaró), Filosofía latinoamericana (Enrique Dussel), Ética (Leonardo Boff), Desarrollo a escala humana (Manfred Max-Neef), entre otras corrientes, prácticas, movimientos sociales, así como grupos originarios, afros, mujeres y minorías sociales y sexuales que se caracterizaron por plantear la diferencia y diversidad de conocimientos y saberes y apostar por epistemes particulares que niegan la pretendida universalidad epistémica euronorteamericana para dar lugar a epistemes propias. Es en este concierto de las Ciencias Sociales latinoamericanas donde "suena con melodía propia" la Pedagogía Crítica. En relación con ello, Mejía plantea:

Hablar de educación y pedagogía crítica desde América Latina es hablar de una práctica social que se ha venido conformando en esta realidad en la segunda mitad del siglo XX y los comienzos de este nuevo milenio [...] se trata de un proceso que nació unido a las dinámicas sociales, políticas y culturales que se desarrollaron en nuestra realidad, buscando una identidad y un sentido propio al ser de acá [...] abierta a las otras culturas, busca concretar un compromiso con las necesidades de transformación de la injusticia en nuestras realidades. (2011, p. 26) [Se añaden las cursivas]

En la misma línea, Bambozzi (2005) plantea que la Pedagogía latinoamericana se encuentra situada en el marco de las alternativas a la colonialidad del saber, su objeto de estudio es la humanización del hombre mediante el proceso educativo, así como el reconocimiento y la denuncia de aquellas prácticas de deshumanización que se han dado a través de la dominación.

Camors (2011) plantea que evolucionar hacia una Pedagogía Social -reflexionada en clave latinoamericana- implica asumir una historia donde las culturas de los pueblos originarios y la síntesis de los pueblos europeos que llegaron a la región aporta elementos específicos y sustanciales para elaborar una nueva pro-

\footnotetext{
6 Como la teoría de la resistencia frente a la dominación, las prácticas culturales desde los aportes de Pierre Bourdieu, las ideas de hegemonía, lucha política y conflicto social de Antonio Gramsci, los planteos de la educación como decolonización, entre otros.
} 
puesta educativa. Por su parte, Krichesky (2011) considera que, en los tiempos que corren en Latinoamérica, el eje de reflexión e intervención de la Pedagogía Social y la educación popular se vincula a la inclusión en tanto derecho a la educación. De acuerdo al autor, estas perspectivas interpelan aquellas hegemónicas que restringen la educación a "la escolarización formal" y marcan nuevas agendas de políticas educativas vinculadas a la accesibilidad, la permanencia, el egreso y la calidad de los aprendizajes y ponen el acento en el sentido social, crítico y emancipatorio de lo educativo, que desbordan una matriz meramente escolar.

Planteado el escenario de emergencia de una educación popular y una Pedagogía Social crítica en nuestro escenario latinoamericano, se hace fundamental explicitar la relación entre ambas.

Para ello, partimos de considerar a la educación popular desde una dimensión amplia y la ubicamos como promotora de un movimiento de liberación, emancipación y concienciación de los pueblos, la cual incide en verdaderos procesos de transformación social, cultural y política y sienta las bases de una sociedad democrática como centro de las relaciones sociales. En este sentido, el educador popular brasileño Carlos Rodrigues Brandao (2014) señala su origen como proyecto revolucionario, cuyo horizonte se fijaba como una megameta: la revolución socialista. Para él, ese horizonte, entonces unificado, hoy se redefine en una pluralidad de metas de mediano plazo, donde la educación popular es un gran paraguas que incluye toda una pluralidad de experiencias, tales como: la emancipación de minorías, mayorías, mujeres, indígenas, movimientos como el LGTB7, la educación ambiental popular, escuelas populares como las del Movimiento Sin Tierra o las de la Vía Campesina, movimientos de negros que luchan por sus tierras, bachilleratos populares, universidades populares, entre otras.

De esta manera, la forma en que se dinamizan estas experiencias de educación popular en los tiempos que corren nos permite pensarlas en una dimensión más concreta que alude a un conjunto de prácticas socioeducativas que -al trascender el escenario escolar- se desarrollan con distintos grupos humanos en situación de opresión, dominación y colonización y atienden a sus propias realidades y situacionalidades, sin despegarse de ese horizonte de transformación y emancipación social.

Por su parte, la Pedagogía Social refiere a una disciplina teórico-práctica en permanente construcción, por eso es dinámica, contextualizada y situada en un tiempo histórico determinado, cuyo objeto es la reflexión, análisis, interpretación, comprensión, explicación y crítica de la educación como práctica social de naturaleza política (que incluye a la educación popular) y atiende a las características que asume, los procesos que promueve, los ámbitos en que se desarrolla, los sujetos educadores y educandos involucrados, los condicionantes que la atraviesan, las

\footnotetext{
7 Se denomina movimiento LGBT al movimiento social y político que lucha contra la discriminación por orientación sexual o identidad de género y que está a favor del reconocimiento de los derechos de las personas lesbianas, gays, bisexuales, transgénero y transexuales, así como de otros colectivos relacionados con la sexualidad.
} 
concepciones filosóficas, antropológicas y ontológicas que subyacen a dichas prácticas, entre otros aspectos.

Esta perspectiva implica, si bien considerar los escenarios formales de educación en sus atravesamientos y condicionamientos sociopolíticos, económicos y culturales, apostar por una pedagogía que revisita un posicionamiento tradicional localizado en la escuela y los trayectos sistemáticos de educación. De esta manera, la pedagogía trasciende muros institucionales para situarse en la comunidad al aproximarse, desde la educación, a los grupos populares. Con ellos, promueve la recuperación de sus propias voces y la injerencia en sus condiciones de existencia, desde la posibilidad de un trabajo participativo, compartido y colectivo.

Volviendo a lo que Mejía (2017) Ilama el cuarto paradigma educativo, este está conformado por una serie de hitos en la memoria histórica del desarrollo de una educación popular así como por toda una tradición de pedagogos que han dado vida a estos procesos. Respecto de estos hitos en la educación popular, deben reconocerse:

- Los aportes de Simón Rodríguez (1769-1854) a la educación popular para los "desaparrados" de la tierra, su influencia en la formación del Libertador Simón Bolívar, a través de sus ideas emancipatorias durante la lucha por la independencia de nuestros pueblos y su anhelo de una Patria Grande para Latinoamérica,

- La Reforma Universitaria iniciada en Córdoba (Argentina) en 1918 y los intentos de construcción de universidades populares latinoamericanas a lo largo de la primera mitad del siglo XX, donde la figura de José Carlos Mariátegui es la promotora de la idea de la universidad como parte de un sistema integral de educación y la incidencia del proyecto político económico como determinantes de las posibilidades revolucionarias de la educación (Mariátegui, 2008),

- La escuela Ayllu de Warisata en Bolivia promovida por Elizardo Pérez y ligada a la sabiduría aymara y quechua,

- Los desarrollos de Paulo Freire desde el movimiento de cultura popular en Recife, a fines de los años cincuenta y durante los sesenta, con los procesos de alfabetización y postalfabetización bajo el nombre de Educación liberadora, Pedagogía del oprimido, Educación emancipadora, Pedagogías crítico-sociales, así como Educación popular (Mejía, 2017; Vogliotti \& Juárez, 2012; Puiggrós, 2010).

En cuanto a la tradición de pedagogos y pedagogas que le han dado vida a este pensamiento de nuestra tierra, debe reconocerse que su mención puede resultar incompleta en tanto muchos de ellos y de ellas no llegaron a ser conocidos, aunque pudieron haber pugnado por verdaderos procesos educativos liberadores. No obstante, hecha esta aclaración, pueden identificarse, en Latinoamérica, a pedagogos y pedagogas destacados que trascendieron con los años por sus experiencias y sus prácticas así como su reflexión en escritos y libros que constituyen verdaderas obras pedagógicas. Algunos de ellos son Juan José Hernández Arregui, Julio Barcos, Olga y Leticia Cossettini, Carlos Vergara, Saúl Taborda y José Arizaga (todos ellos de Argentina); Simón Rodríguez (Venezuela); José Carlos Mariátegui y Raul Haya de la Torre (ambos de Perú); José Martí (Cuba); Elizardo Pérez (Bolivia) y Paulo Freire (Brasil), entre tantos otros pensadores que 
-incluso sin ser pedagogos- influyeron en el desarrollo y crecimiento de este campo de conocimiento en pos de la transformación de la educación para la generación de una sociedad más justa, humana e inclusiva.

En este escenario, tampoco es posible dejar de hacer mención a los desarrollos de Paulo Freire $^{8}$ (1991-1997) al campo de la educación popular y la pedagogía crítica, dado que ha generado uno de los aportes más valiosos al campo pedagógico latinoamericano. Esto llevó a autores como Torres Carrillo (2009) a considerar que Freire no es patrimonio solo de las pedagogías alternativas, sino del pensamiento emancipador del mundo, ya que su legado constituye un referente ineludible siempre que se pretenda la reconstrucción del pensamiento emancipador ${ }^{9}$. Son sus desarrollos de una educación liberadora y una pedagogía crítica los que van a hacer un quiebre respecto de los modelos hegemónicos, bancarios y extensionistas en educación formal.

Freire pudo reconocer el problema de su época al identificarlo en la hegemonía de un modelo económico capitalista, generador de explotación humana, dominación, pobreza, y su correlato, en lo socioeducativo, lo que da cuenta de una realidad brasileña con los índices de analfabetismo más altos de su historia. Para su abordaje político y metodológico, a través del Movimiento de Cultura Popular, Freire elabora una propuesta que parte de la aproximación a los grupos populares para investigar y conocer con ellos sus problemas, demandas, necesidades, intereses, sus propias palabras y temas de su época, y con base en ello, construir las experiencias de educación problematizadora para promover procesos de alfabetización (investigación de la palabra generadora) y postalfabetización (investigación del tema generador) y concebir la naturaleza política de la educación y la posibilidad de transformación de las estructuras sociales injustas a través de su realización. La "educación liberadora" es entendida desde su concepción como un fin en sí mismo y un medio para la construcción de poder popular en pos de la transformación social.

Monteiro Machado (2011) plantea que, en 1960, Freire inicia la teoría y práctica de una Pedagogía Social y, a pesar de no haber utilizado esa denominación para definir su trabajo, es reconocido como una referencia brasileña e internacional de la Pedagogía Social y el análisis de sus experiencias de educación popular es un camino que comienza a trazarse en pos de la construcción de una Pedagogía Social en nuestra región.

\footnotetext{
8 Rosa María Torres (1985, p. 138) aclara que, en sus primeros escritos, Freire no hablaba de educación popular, sino de educación liberadora, educación para la libertad, educación para la democracia, educación democrática, educación problematizadora, educación cultural popular, educación crítica, educación dialógica. El mismo Carlos Rodríguez Brandao recuerda: "Trabajábamos en eso que sería un nuevo sentido de la educación. Buscábamos un nombre a eso. El primer nombre no fue educación popular. Educación popular es un nombre tardío en ese sentido: el primer nombre fue educación liberadora, el primer nombre que incluso le dio el mismo Paulo Freire" (Ventura, 2007) [Se añaden las cursivas].

9 Torres Carrillo (2009) plantea que, en la última década, algunos pedagogos latinoamericanos (João Francisco de Sousa, Carlos Nuñez, Alfredo Ghiso, Oscar Jara, Lola Cendales, Pedro Pontual, Moacir Gadotti) y de otras latitudes (Mc Claren, Giroux, Carlos Alberto Torres, Walsh) han retomado y recreado algunas de las ideas de Freire en torno a temáticas como la interculturalidad, la ética pedagógica, el diálogo, el compromiso, la formación de educadores y las pedagogías críticas.
} 


\section{3. Últimas reflexiones}

Este artículo ha intentado hacer un recorrido contextualizado por los dos grandes paradigmas que han impactado en el campo de la Pedagogía Social y trazar un mapeo general que trata de articular un entramado coherente que ofrezca claridad para la comprensión del devenir paradigmático en el campo pedagógico y su influencia en el actual escenario de conformación de una pedagogía latinoamericana, entendida como un paradigma alternativo en sí mismo y propio de nuestra región. Si bien puede reconocerse que lo planteado hasta aquí favorecerá el despunte de nuevos temas y problemas en el interior del campo pedagógico, susceptibles de ser profundizados y ampliados en futuros análisis, esta instancia de cierre intenta centrarse en dos aspectos: por un lado, comprender la necesidad de atender a las particularidades que asumen los procesos educativos populares en la actualidad, y, por el otro, partiendo de este escenario, aportar a una dilucidación crítica de esa realidad desde un posicionamiento sustentado en el paradigma pedagógico latinoamericano.

Respecto al escenario actual de la educación popular, tal como plantea Krichesky (2011), debe reconocerse que este no es el mismo que el de los años setenta. Si bien, tras el paso de gobiernos progresistas en algunos países de la región, pudo reconocerse cierta crisis de hegemonía del modelo neoliberal que favoreció un mayor protagonismo de movimientos sociales, la inserción de las ONG en políticas públicas, el desarrollo del poder local y distintos progresos a nivel de derechos humanos en todos los planos, por estos días, la situación es diferente. Se vivencia un recrudecimiento de la tendencia neoliberal y neoconservadora en las estructuras de poder del Estado, que exige a la educación popular ${ }^{10}$ y a la pedagogía crítica latinoamericana el desafío de generar nuevas praxis socioeducativas desde los grupos y movimientos sociales, promotoras de conciencia crítica y de acción ante estas nuevas coyunturas sociales y políticas que trazan un horizonte incierto -en generalpara la sociedad toda, pero -en particular- para los grupos populares, por ser el blanco de las embestidas de las decisiones, acciones y omisiones de estos grupos de poder. El escenario brevemente planteado exhorta, más urgente que tarde, a la asunción de un posicionamiento claro en la realidad de nuestra región. Se considera así que los aportes de la Pedagogía Social crítica latinoamericana pueden interpelar esos lugares en que los educadores, pedagogos y educandos se colocan en rela-

10 Krichesky (2011, p. 63) plantea las particularidades que asumen en los países latinoamericanos las prácticas autodefinidas como de educación popular y advierte que muchas de ellas participan de procesos de diálogo con el Estado desde intencionalidades vinculadas a demandar su gradual legitimación, mientras que otras se valen de los resquicios o intersticios del sistema con el propósito de lograr cierto financiamiento como instituciones educativas, como es el caso de un grupo numeroso de jardines comunitarios y bachilleratos populares. Puede agregarse como ejemplo de ello el caso del Bachillerato Popular de Jóvenes y Adultos del IMPA -Industrias Metalúrgicas y Plásticas Argentinas-, una experiencia de más de doce años que da cuenta de cómo las organizaciones sociales pueden autogestionar sus propios espacios educativos desde una propuesta esperanzada que sienta las bases para la lucha contra los efectos expulsivos de un neoliberalismo que se recrudece con el paso de los años (Colectivo de IMPA, 2016). No obstante, el autor reconoce otras experiencias que resisten taxativamente la posibilidad de ser parte del sistema y se sostienen con esfuerzos y recursos propios, desde prácticas autogestivas. Tanto en un caso como en otro, el autor reconoce que resultan innegables y valiosos los aportes de la Educación Popular al campo de la Pedagogía Social, a las cuestiones de la inclusión y al derecho a la educación en términos de recuperar la perspectiva emancipadora, crítica y política del acto de educar. 
ción a dimensiones vinculadas a qué tipo de prácticas educativas se intentan promover, en relación a qué concepción de ser humano y para qué sociedad. Precisar estas cuestiones implica asumir un posicionamiento político.

Respecto al primer aspecto, el pensamiento pedagógico crítico latinoamericano parte de considerar al ser humano como un ser inacabado que "está siendo" en un contexto situado, en tiempo y espacio determinado, un ser en devenir que transforma el mundo a partir de su inserción en la realidad, que toma injerencia en ella desde la toma de decisiones para modificar sus condiciones de existencia concretas, y que, para ello, se implica en la crítica y la reflexión colectiva sobre su tiempo histórico, del que se considera protagonista. De esta manera, el ser humano se constituye en un ser capaz de revisitar su pasado para comprender su presente y proyectar un futuro realizable desde la utopía de la liberación. Asimismo, el ser humano es un ser integral, atravesado por las dimensiones de lo físicocorporal, lo psíquico-emocional, lo ambiental y lo espiritual, como aquello que emerge por el contacto entre el psiquismo, la cultura y el interrogante ¿por qué vivimos? Se trata de dimensiones engarzadas a su condición social de ser protagonista de su tiempo histórico junto con otros, en unas circunstancias socioculturales e históricas determinadas.

En cuanto a la concepción de realidad, el pensamiento pedagógico crítico latinoamericano entiende que esta no es inexorable ni está determinada, sino que presenta distintos condicionamientos de las estructuras políticas, económicas, sociales y culturales y que esto puede ser transformado por la acción del sujeto. La realidad social "está siendo", es devenir y, en ella, nada está dado ni es inmodificable, sino que es susceptible de transformaciones por la misma acción, crítica y compromiso de hombres y mujeres.

Emerge así una concepción de conocimiento propia del pensamiento pedagógico latinoamericano desde la cual se lo concibe como una construcción humana continua que supone una relación entre el sujeto que conoce y su propia realidad como objeto de conocimiento que lo implica en la reflexión y acción constante en torno a dicha realidad.

La explicitación del propio posicionamiento por parte de los actores del campo de la educación -pedagogos, educadores y educandos- se torna una tarea éticopolítica fundamental, dado que permite dilucidar a favor de qué, de quién y de quiénes se reflexiona, se enseña y aprende $y$, eventualmente, en contra de qué, quién y quiénes se realizan estos procesos ${ }^{11}$. En este sentido, los aportes que la

\footnotetext{
11 Reflexionar sobre un pensamiento pedagógico latinoamericano que se pronuncia en "contra de" refiere a una toma de conciencia político-pedagógica sobre la denuncia a un sistema económico, material, simbólico y capitalista que "forma" desde el control de su fuerza penetrante y persuasiva, lo que genera y mantiene opresión, dominación, exclusión y marginación de grupos humanos. Se plantea así la necesidad de formar, educar y reflexionar sobre estos procesos en contra de una modelación orientada a una servidumbre voluntaria, a una libertad individualista, a una competición económica global a la que solo le importan productores y consumidores, cuya intencionalidad es edificar un perfil identitario y biográfico bajo el lema del mayor éxito posible, lo que promueve, como dice Anna Piussi (2009), una autonomía atomística que implica una autosuficiencia ilusoria, una vida esclava a los mecanismos reguladores del mercado, aspectos sobre los que una pedagogía latinoamericana crítica asume la tarea, por acción y reflexión, de pronunciarse explícitamente en contra.
} 
Pedagogía Social latinoamericana realiza a la definición de un posicionamiento socioeducativo crítico son inagotables y suponen una apuesta a un presente latinoamericano sustentado en un pasado rico en experiencias, tradición de pedagogos y un carácter eminentemente progresista de esta pedagogía, en tanto supone una implicancia política en términos críticos, de reflexión y acción sobre la realidad para transformarla. Así entendida, la Pedagogía Social de ayer, de hoy y de todas las apuestas que vendrán será "praxis" (Freire, 1973, 1990).

\section{Referencias bibliográficas}

Bambozzi, E. (2005). Escritos pedagógicos. Córdoba: Ediciones del Copista.

Barrios, M. A. (2011). El latinoamericanismo educativo en la perspectiva de la integración regional. Buenos Aires: Biblos.

Bazán Campos, D. (2008). El Oficio del Pedagogo. Aportes para la construcción de una práctica reflexiva en la escuela. Buenos Aires: HomoSapiens.

Borzese, D., Costas, P. \& Wanger, L. (2011). Educación social y políticas públicas. La educacio?n social en la Argentina ante el desafío de justicia educativa. En M. Krichesky (Ed.), Cuadernos de trabajo \# 2: Pedagogía Social y educación popular. Perspectivas y estrategias sobre la inclusión y el derecho a la educación (pp. 91101). La Plata: UNIPE Editorial Universitaria.

Camors, J. (2011). Hacia la pedagogía que la educación requiere, aquí y ahora. En M. Krichesky (Ed.), Cuadernos de trabajo \# 2: Pedagogía Social y educación popular. Perspectivas y estrategias sobre la inclusión y el derecho a la educación (pp. 9-21). La Plata: UNIPE Editorial Universitaria.

Caride, J., Gradaílle, R. \& Caballo, M. (2015). De la pedagogía social como educación, a la educación social como Pedagogía. Revista Perfiles Educativos, $X X X V I I(148), 4-11$.

Colectivo de IMPA. (2016). Praxis Política y Educación Popular. Apuntes en torno a una pedagogía emancipatoria en las aulas del Bachillerato IMPA. Buenos Aires: Naranjo en Flor, editorial Rioplatense.

Díaz, E. (2007). Entre la tecnociencia y el deseo. La construcción de una epistemología ampliada. Buenos Aires: Biblos.

Espiga, E., López, W. \& Morales, M. (2012). Educación social en Uruguay: apuntes para pensar la formación. Revista Pedagógica, 2, 88-105.

Freire, P. (1973). Pedagogía del Oprimido. Buenos Aires: Siglo XXI.

Freire, P. (1990). La naturaleza política de la educación. Cultura poder y liberación. Buenos Aires: Paidós. 
Freire, P. (1993). Pedagogía de la esperanza. México: Siglo XXI.

Gvirtz, S., Grinberg, M. \& Abregú, V. (2011). La educación de ayer, hoy y mañana. El $A B C$ de la pedagogía. Buenos Aires: Aique.

Klimovsky, G. (1994). Las desventuras del conocimiento científico. Una introducción a la epistemología. Buenos Aires: A-Z Editora.

Klimovsky, G. \& Hidalgo, C. (1998). La inexplicable sociedad. Cuestiones de epistemología de las Ciencias Sociales. Buenos Aires: A-Z Editora.

Krichesky, M. (2011). Pedagogía Social y educación popular. Tensiones y aportes sobre el derecho a la educación. En M. Krichesky (Ed.), Cuadernos de trabajo \# 2: Pedagogía Social y educación popular. Perspectivas y estrategias sobre la inclusión y el derecho a la educación (pp. 55-66). La Plata: UNIPE Editorial Universitaria.

Kuhn, T. (1962). La estructura de las revoluciones científicas. México, D.F.: Fondo de Cultura Económica.

Mariátegui, J. C. (2008). La reforma universitaria: desafíos y perspectivas noventa años después. Buenos Aires: CLACSO. Recuperado el 2 de febrero de 2018, de http://biblioteca.clacso.edu.ar/clacso/gt/20101109083911/22mariate.pdf.

Mejía, M. R. (2011). Educaciones y pedagogías críticas desde el sur. Cartografías de la educación popular. Lima: CEAAL.

Mejía, M. R. (2017). Educaciones y pedagogías críticas desde el sur. Cartografías de la educación popular. Buenos Aires: La Crujía.

Mignolo, W. (2007). El pensamiento decolonial: desprendimiento y apertura. Un manifiesto. En S. Castro Gómez \& R. Grosfoguel (Eds.), El giro decolonial. Reflexiones para una diversidad epistémica más allá del capitalismo global (pp. 2546). Bogotá: Siglo del Hombre Editores.

Monteiro Machado, E. (2011). Encuentros y desencuentros entre la Pedagogía Social y la educación popular en América latina: "el caso de Brasil". En M. Krichesky (Ed.), Cuadernos de trabajo \# 2: Pedagogía Social y educación popular. Perspectivas y estrategias sobre la inclusión y el derecho a la educación (pp. 37-53). La Plata: UNIPE Editorial Universitaria.

Núñez, V. (2007). Pedagogía Social: un lugar para la educación frente a la asignación social de los destinos. Recuperado el 15 de febrero de 2018, de http:// www.porlainclusion.educ.ar/documentos/Violeta_N_Pedagogia_Social.pdf.

Pérez Serrano, G. (2002). Origen y evolución de la Pedagogía Social. Pedagogía Social. Revista interuniversitaria, 9(2), 193-231.

Piussi, A. M. (2009). Posibilidad de una escuela de libertad. En L. Boff, V. Ferrer, F. Gutiérrez, H. Maturana \& A. M. Piussi (Eds.), Figuras y pasajes de la complejidad en la educación. Experiencias de resistencia, creación y potencia (pp. 1-20). Valencia: Instituto Paulo Freire. 
Puiggrós, A. (1990). Historia de la Educación Argentina. Tomo 1: Sujetos, disciplina y currículum en el sistema educativo argentino. Buenos Aires: Galerna.

Puiggrós, A. (2010). De Simón Rodríguez a Paulo Freire. Educación para la integración Iberoamericana. Buenos Aires: Colihue.

Puiggrós, A. (2017). Adiós, Sarmiento. Educación pública, Iglesia y mercado. Buenos Aires: Colihue.

Quintar, E. [Centro de Estudios Avanzados en Niñez y Juventud] (2018, 23 de agosto). La cuestión epistemológica como mirada del mundo de la vida [Archivo de video]. Recuperado el 21 de marzo de 2019, de https://www.youtube.com/ watch $? v=x X B f E J D \_f A Y \& f e a t u r e=y o u t u . b e$.

Rodrigues Brandao, C. (2014, 2 de enero). La educación popular es un gran paraguas. Entrevista realizada por Laura Vales. Página 12, sección El país [en línea]. Recuperado el 29 de marzo de 2019, de https://www.pagina12.com.ar/diario/elpais/ 1-236859-2014-01-02.html

Saviani, D. (1982). Las teorías de la Educación y el problema de la marginalidad en América Latina. Revista de Estudios e Investigación, 42, 1-12.

Segato, R. (2018). Contra-pedagogías de la crueldad. Buenos Aires: Prometeo.

Torres Carrillo, A. (2009). Educación popular y paradigmas emancipares. Revista Pedagogía y Saberes, 30, 19-32.

Torres, R. M. (1985). Sobre educación popular: Entrevista a Paulo Freire. En R. M. Torres, Educación popular: un encuentro con Paulo Freire (pp. 117-153). Sao Paulo: Biblioteca digital CREFAL.

Vélez, G. (2002). Aprender en la universidad. La relación del estudiante universitario con el conocimiento. Materiales de aula SIAT. Comité Editor de la Facultad de Ciencias Humanas. Universidad Nacional de Río Cuarto. Recuperado el 15 de marzo de 2018, de http://www.siat.unrc.edu.ar.

Ventura, T. (2007). Paulo Freire Contemporáneo [Video documental]. Recuperado el 20 de abril de 2020, de https://www.youtube.com/watch? $v=5$ s2Fmtr3FN8\&t=69s.

Vogliotti, A. \& Juárez, M. P. (2012). Glosario Freireano. Significados para comprender y recrear la teoría freireana. Córdoba: Jorge Sarmiento Editor.

Vogliotti, A. (2001). Relación educativa como instancia de conocimiento: ¿es posible la compatibilidad de las perspectivas constructivista y crítica? Río Cuarto: EFUNARC.

Vogliotti, A. (2015). Epistemología y pedagogía: ¿La realidad es o se hace? ¿La pedagogía es o la construimos? [Material inédito de uso interno]. Cátedra de Pedagogía, Departamento de Ciencias de la Educación. Facultad de Ciencias Humanas, Universidad Nacional de Río Cuarto.

Vogliotti, A. (2016). Paradigmas epistemológicos en las Ciencias Sociales. [Material inédito de uso interno]. Módulo: Pensamiento Crítico y Alfabetización, Facultad de Ciencias Humanas, Secretaría Académica, UNRC. 